\title{
Educación en Colombia: el enfoque por competencias y la "glocalización"
}

\author{
Education in Colombia: \\ an Approach for Competences and "Glocation"
}

Maurizio Milazzo R. ${ }^{1}$

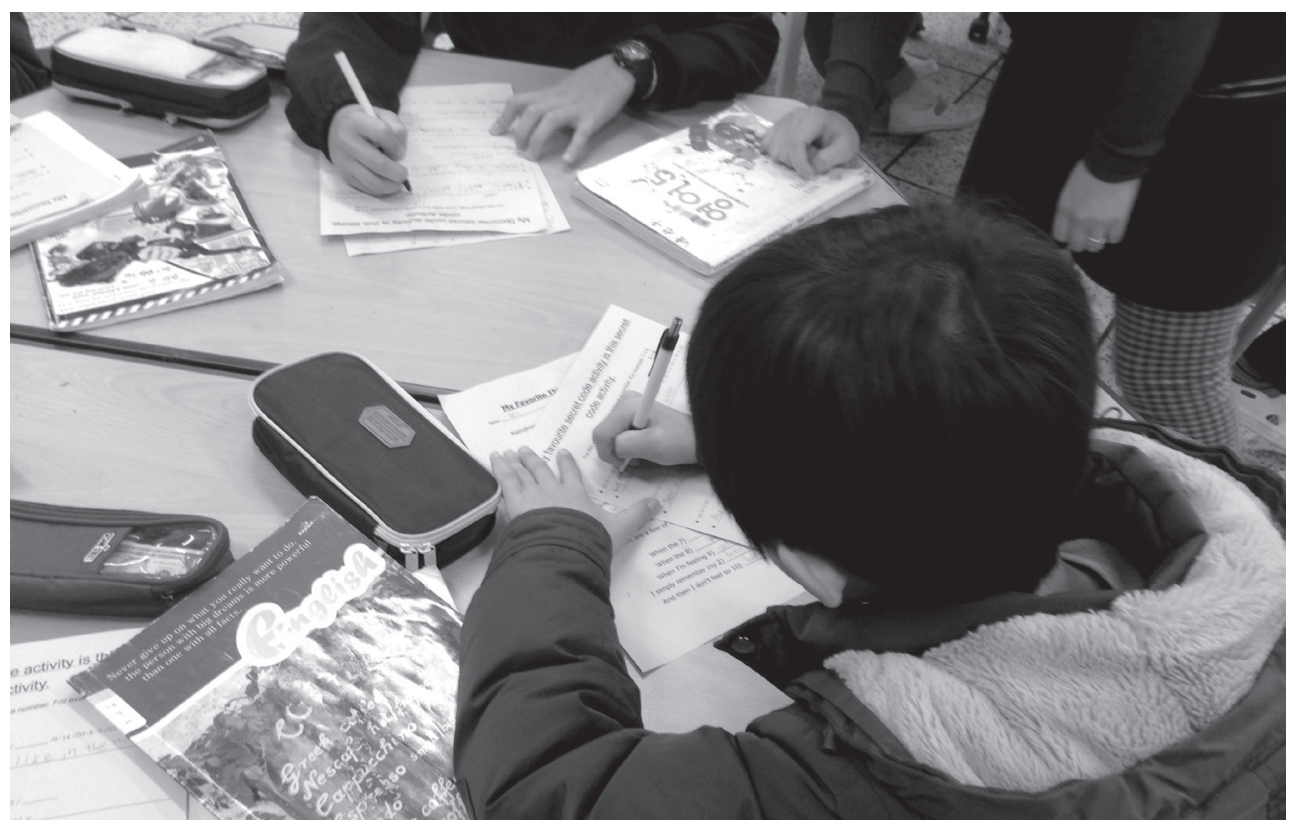

\section{Resumen}

En la dicotomía entre universalismos y localismos, la cultura occidental hegemónica eurocentrista ahora llega a sus límites. En este contexto, y dado que no se puede pensar en un solo patrón cultural global en el que quepan todas las formas plurales de cultura, emerge la necesidad de educar tanto sobre postulados universales foráneos, como sobre saberes locales. El enfoque particular de competencias acogido y promocionado por el Ministerio de Educación Nacional de Colombia logra retraídamente armonizar estos extremos de globalidad y localidad.

Palabras clave: globalidad, localidad, cultura occidental hegemónica, universalismos. competencias y la "glocalización". Revista Papeles, 10(20), 101-108.

Fecha de recibido: agosto 152018 • Fecha de aceptación: noviembre 10 de 2018

1 Profesor de la Universidad Externado de Colombia. Correo electrónico: mauricio.milazzo@uexternado.edu.co 


\begin{abstract}
In the dichotomy between universals and localisms, hegemonic Western Eurocentric culture has now reached its limits. In this context, and given the fact that we cannot think of one global cultural pattern to which all plural forms of culture can belong, the need arises to educate towards these proposed foreign universals in the same way as local knowledge. The particular approach of competencies adopted and promoted by the Colombian National Ministry of Education has begun to cautiously harmonize these extremes of globalness and localness.
\end{abstract}

Keywords: hegemonic, Western Eurocentric culture, hegemonic, universals.

\title{
Introducción
}

En el presente texto se resalta mucho la importancia de tener una consciencia cosmopolita, pero sin dejar de lado las especificidades de lo local y sin olvidar la importancia pragmática del aquí y el ahora (hic et nunc).

De esta suerte de imperativo categórico del presente puede emerger el interrogante: ¿cómo se articulan estos dos extremos de esta dicotomía? Pues bien, se articulan en la tensión permanente entre universalidad y particularidad, es decir, entre unos postulados que tienen una pretensión de validez mundial, global, y otros que se circunscriben a una validez nacional o regional: local.

La reflexión sobre uno de los extremos de esta dicotomía, la globalización, ya ha estado presente en el ámbito de las organizaciones educativas. En efecto, en la UNESCO se suscita una quaestio sobre el papel de las organizaciones en torno a esta globalización, más allá incluso de las esferas de la educación y la cultura; así se plantea en el informe a la UNESCO de la Comisión Internacional sobre la Educación para el siglo XXI:

La interdependencia planetaria y la mundialización son fenómenos esenciales de nuestra época. Actúan ya en el presente y marcarán con su impronta el siglo XXI. Hoy hacen ya necesaria una reflexión global -que trascienda ampliamente los ámbitos de la educación y la culturasobre las funciones y las estructuras de las organizaciones internacionales. (Delors, 1996, p. 31)

En este mismo escenario de deliberación en torno a la educación - la UNESCO_, aparece igualmente la reflexión sobre la identidad expresión par excellence de lo local一, así:

La utopía orientadora que debe guiar nuestros pasos consiste en lograr que el mundo converja hacia una mayor comprensión mutua, hacia una intensificación del sentido de la responsabilidad y de la solidaridad, sobre la base de aceptar nuestras diferencias espirituales y culturales. Al permitir que todos tengan acceso al conocimiento, la educación tiene un papel muy concreto que desempeñar en la realización de esta tarea universal: ayudar a comprender el mundo y a comprender al otro, para así comprenderse mejor a sí mismo. (Delors, 1996, [Negrilla fuera del texto original])

En el referido informe se entiende que algunos ítems pueden tener validez local y a la vez universal, los cuales pueden servir de norte. En efecto, se dice: "la Comisión trató de razonar dentro de un marco prospectivo dominado por la mundialización, de seleccionar las buenas preguntas que se nos plantean a todos, y de trazar algunas orientaciones validas a nivel nacional y a escala mundial" (Delors, 1996, p. $31)$. 
A este respecto, el sociólogo neoyorkino Immanuel Wallerstein nos ilustra sobre cómo la relación entre lo universal y lo particular es siempre dinámica, y nos muestra, así mismo, cómo esta relación y la primacía de uno de los extremos de ella puede llegar a tener efectos epistemológicos.

En efecto, Immanuel Wallerstein plantea que no existen verdades universales, pues estas son simplemente particularismos que se pretenden universales. Dice Wallerstein:

En los últimos años ha habido un firme repudio al concepto de cultura global (...) El argumento fundamental es que la aserción de verdades universales, dentro de las que se incluyen las normas universales, es una $<<$ metanarrativa $>>$ $\mathrm{o}<<$ narrativa maestra $>$ (una narrativa global) que representa una ideología de grupos poderosos dentro del sistemamundo. (Wallerstein, 2004, p. 124)

En este orden de ideas, se puede decir que todas las ideas emergen inicialmente como locales, y sucede que por múltiples razones, principalmente geopolíticas (de poder), entre otras, algunas se expanden y logran tener una cobertura o validez global. Es el caso de muchas expresiones de la cultura europea, como las lenguas o la idea de Estado, que se expandieron por el mundo, y por ello vemos en África y en América, tanto formas republicanas de organización política, como lenguas europeas - el inglés, el español, etcétera.

Ahora bien, en el mundo postmoderno esta hegemonía de las formas culturales europeas, esta pretensión de validez universal de estas expresiones culturales ha empezado a ser cuestionada. En efecto, Vattimo plantea cómo, con el fin del colonialismo,

los pueblos $<<$ primitivos $>>$, los así llamados, colonizados por los europeos en nombre del buen derecho de la civilización $<<$ superior $>>$ y más desarrollada, se han rebelado y han vuelto problemática de hecho una historia unitaria centralizada. El ideal europeo de humanidad se ha manifestado como un ideal más entre muchos otros, no necesariamente peor, pero que no puede pretender, sin violencia, el derecho de ser la esencia verdadera del hombre, de todo hombre. (Vattimo, 1994, p. 12)

Varios autores, entre ellos el mencionado Immanuel Wallerstein y el pensador colombiano Enrique Ferrer, consideran que si bien puede ser problemática la idea de la validez universal de ciertos postulados, no por ello se puede cerrar la puerta a que existan ciertas normas universales, con varios propósitos, por ejemplo, para la materialización de la idea de democracia (Wallerstein, 2004 y Ferrer, 2009).

En esta dicotomía entre lo local y lo global, un modelo de postulado con pretensión de validez universal par excellence es la idea de los Derechos Humanos. Sin embargo, como expresión de un extremo de esta mencionada dicotomía no está exento de críticas, pues existen dificultades para postular unos derechos que sean valederos para todas las comunidades nacionales por igual, es decir, que sean globales y no entren en contradicción con expresiones normativas nacionales o locales (Kymlicka, 2003).

En efecto, el filósofo político canadiense Will Kymlicka nos ilustra sobre cómo la pluralidad

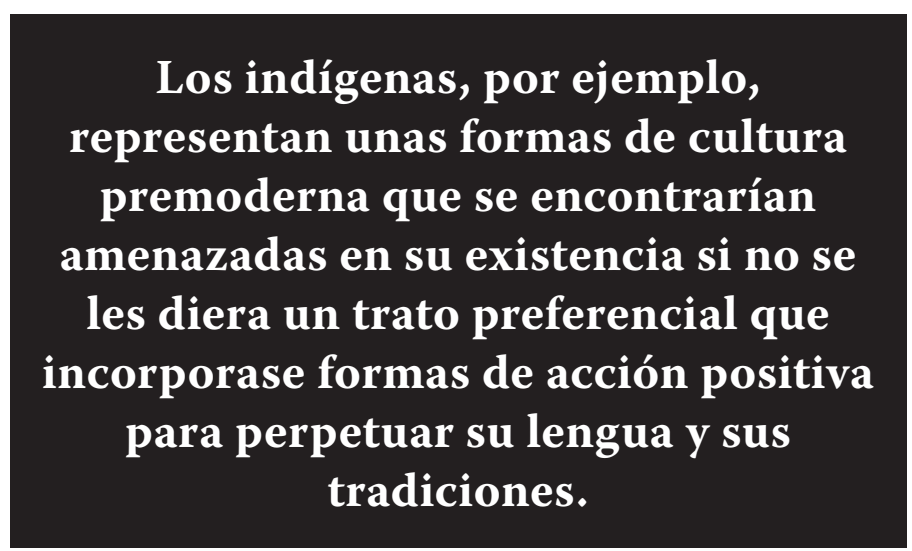


de formas de asumir los temas principales de la vida, como la familia, el poder o la sexualidad, no pueden estar definidas de antemano por unos postulados que se pretendan globales; y plantea:

Algunos portavoces indígenas dirían que el hecho de esperar que los gobiernos indígenas deban ser democráticos viola la $<<$ integridad $>>$ de las culturas indígenas, que pueden tener tradiciones centenarias de gobierno no democrático. De forma similar, las normas de la igualdad sexual podrían entrar en conflicto con tradiciones profundamente arraigadas (Kymlycka, 2003, p. 181).

En este orden de ideas, los procesos de descolonización que se consolidaron en todo el mundo en el último siglo dan cuenta de la presencia de formas culturales autóctonas que perviven a la implantación de las formas culturales foráneas, y estas otras expresiones culturales no tienen por qué tener una legitimidad menor a las impuestas por el colonizador. En efecto, no podemos olvidar que en la mayoría de sociedades de origen colonial en el mundo (y la mayoría de sociedades son postcoloniales) existen formas plurales de cultura, no las formas eurocéntricas monolíticas que se han impuesto. En consecuencia, no se puede pensar en un concepto único de cultura, y menos en una cultura global; no se puede pensar en un solo patrón o un canon en el que quepan todas las formas plurales de cultura.

Los indígenas, por ejemplo, representan unas formas de cultura premoderna que se encontrarían amenazadas en su existencia si no se les diera un trato preferencial que incorporase formas de acción positiva para perpetuar su lengua y sus tradiciones. Para Kymlicka, este tratamiento diferenciado es el tratamiento adecuado, tomando en cuenta estas consideraciones (Kymlicka, 2003). En nuestro país, un país multicultural por antonomasia, con más de 80 etnias $^{2}$, este tratamiento diferenciado es acogido por la Constitución Política de 1991, que reconoce la multiculturalidad existente en su artículo $7^{\circ}$, "El Estado reconoce y protege la diversidad étnica y cultural de la Nación Colombiana."; y en su artículo $10^{\circ}$, la misma Constitución establece este mismo tratamiento en la educación local, al plantear que: "El castellano es el idioma oficial de Colombia. Las lenguas y dialectos de los grupos étnicos son también oficiales en sus territorios. La enseñanza que se imparta en las comunidades con tradiciones lingüísticas propias será bilingüe".

En este marco conceptual debemos mirar de qué manera las políticas públicas de educación en Colombia hacen posible que la articulación entre lo local y lo universal (o con pretensiones universalistas) no hagan desvanecer la riqueza cultural local, al ilustrar e instruir sobre saberes con vocación global.

Prima facie podemos ver que en Colombia aparecen propuestas que implican la continuidad del eurocentrismo con su pretensión universalista, pero también coexisten elementos de ruptura y de revalorización de los usos y saberes locales.

Miraremos para el efecto de este estudio el enfoque de "educación por competencias", y algunas competencias ciudadanas en particular. Comencemos por puntualizar que las competencias constituyen parámetros de aquello que los estudiantes deben saber y saber hacer al término de su formación académica en el sistema educativo (Ministerio de Educación Nacional de Colombia, 2006). Ahora bien, al observar la idea misma de "educación por competencias", vemos que este enfoque constituye un cierto estándar educativo que tiene pretensión universalista, y que de hecho ha sido instaurado y está siendo utilizado y desarrollado en muchos países. Sin embargo,

\footnotetext{
2 Nota: 84 etnias registradas en la Resolución 166
} del Ministerio de Educación Nacional. 
esta idea de "competencias" responde a una visión pragmatista y utilitarista de la educación, de raigambre primariamente anglosajona, independientemente de las hibridaciones y aclimataciones múltiples que pueden haberla enriquecido. En efecto, este enfoque es un legado de los empiristas norteamericanos, especialmente de John Dewey, pues antes de su conceptualización sobre la educación no existía en Estados Unidos - su país de origen y objeto de estudio- un sistema educativo público consolidado, pues la educación integral era un privilegio de pocos en ese momento allí (Dewey, 2004). En este orden de ideas, este enfoque por competencias es una de las creaciones locales, particularista, que se globalizó y ha alcanzado el satus de saber universal.

Ahora bien, más allá de las bondades o falencias del enfoque mismo, hemos de mirar en concreto algunas de las competencias educativas propuestas por el Ministerio de Educación Nacional de Colombia (MEN), como política pública, y examinar en qué medida posibilitan una articulación entre lo local y lo global, articulación que sea enriquecedora de la experiencia educativa de los estudiantes. Esta experiencia "pluricultural" constituye un agregado enriquecedor de la formación integral del estudiante, y en ámbitos multiculturales es un requisito sine qua non de la educación. En este sentido se ha manifestado la Unión Europea al definir un estándar común de educación en lenguas, dentro del llamado Marco Común Europeo de Lenguas, en uno de cuyos documentos se expresa:

En la competencia cultural de una persona, las distintas culturas (nacional, regional, social) a las que ha accedido esa persona no coexisten simplemente una junto a otra. Se las compara, se las contrasta e interactúan activamente para producir una competencia pluricultural enriquecida e integrada. (Ministerio de Educación, Cultura y Deporte, España, 2002)
En este orden de ideas, y retomando el tratamiento diferenciado que, como dijimos, ha asumido la Constitución Política de 1991, vemos que igualmente lo consagran algunas de las competencias propuestas por el Ministerio, aunque de manera atenuada, como, por ejemplo, aquella que dice: "Reconozco que pertenezco a diversos grupos (familia, colegio, barrio, región, país, etc.) y entiendo que eso hace parte de mi identidad" (MEN, 2006). En efecto, al enseñársele al estudiante que existen diferentes tipos de pertenencias expresadas en familias, grupos o regiones plurales, se le está enseñando la existencia de la multiculturalidad en Colombia, y se le está enseñando también la importancia de la identidad como expresión de esa pertenencia. La importancia de educar en la identidad, en la pertenencia, había sido ya resaltada por John Dewey, quien, haciendo una analogía con la renovación de la vida biológica de las especies, explica cómo también se da una renovación de los grupos sociales mediante el proceso educativo que forma a los individuos en los valores, saberes y prácticas sociales. Dice Dewey:

La continuidad de toda experiencia, mediante la renovación del grupo social, es un hecho literal. La educación, en su sentido más amplio, es el medio de esta continuidad de la vida. Cada uno de los elementos constitutivos de un grupo social, tanto en una ciudad moderna como en una tribu salvaje,

"El castellano es el idioma oficial de Colombia. Las lenguas y dialectos de los grupos étnicos son también oficiales en sus territorios. La enseñanza que se imparta en las comunidades con tradiciones lingüísticas propias será bilingüe”. 
nace inmaduro, indefenso, sin lenguaje, creencias, ideas ni normas sociales. Cada individuo, cada unidad de portadores de la experiencia vital de su grupo desaparece con el tiempo. Y sin embargo, la vida del grupo continúa. (Dewey, 2004, p. 14 [Negrilla fuera del texto original])

En este orden de ideas, la educación pluricultural es necesaria para la pervivencia de las culturas, sean vernáculas o contemporáneas, toda vez que sin educación la cultura perece, pues la educación es una herramienta indispensable para preservar lo construido, lo cultivado: la cultura. La educación es, para decirlo en términos spinozianos, el instrumento para lograr el conatus: la preservación en la existencia, de una cultura dada.

Ahora, si pensamos en los muchos grupos minoritarios existentes en el país, en concreto en las etnias indígenas, vemos que de conformidad con los tratados internacionales uno de sus derechos es el de la <<identidad cultural $>>$. Es de anotar que esta consciencia de la pertenencia y de la identidad es importante para todos, independientemente de su origen nacional, étnico o regional, pues la libertad - uno de los postulados de las democracias-, bien se puede entender como esa tensión que existe entre pertenencia y desasimiento (Vattimo, 1994), pertenencia a un grupo o familia, y desasimiento al explorar el mundo e intercambiar con otros (otros grupos, otras familias, etc.); pertenencia a lo local y desasimiento al aprehender lo no local: lo global.

Otra competencia que da cuenta de la multiculturalidad la plantea el Ministerio, así: "Comprendo que existen diversas formas de expresar las identidades (por ejemplo, la apariencia física, la expresión artística y verbal, y tantas otras...) y las respeto" (MEN, 2006). Esta competencia comunicativa es enriquecedora para el estudiante al permitirle expresar su identidad en formas plurales, y entender que los otros pueden y deben también expresar su identidad en múltiples formas; enseñanzas estas que permiten vehicular la identidad propia, la forma autóctona de percibir y de estar en el mundo.

Este respeto y reconocimiento de la multiculturalidad y de las identidades que ella implica son conditio sine qua non del desarrollo de la personalidad de los individuos y condición de supervivencia de las colectividades, toda vez que como expresa Kymlicka, "Abandonar la propia cultura, aunque es posible, se considera más bien como renunciar a algo a lo que razonablemente se tiene derecho" (Kymlicka, 1995, p. 125). Y aún desde una perspectiva globalizadora, universalista, se entiende el derecho a permanecer y replicar la cultura y el deber de los otros a respetar esa posibilidad, y así lo contempla también el pensador canadiense, quien dice:

Este no es un supuesto acerca de los límites de las posibilidades humanas, sino acerca de las expectativas razonables. Pienso que la mayoría de los liberales han aceptado implícitamente este supuesto acerca de las legítimas expectativas de la gente a permanecer en sus culturas (Kymlicka, 1995, p. 125).

En este mismo sentido, la jurista peruana Columba del Carpio plantea una forma de ciudadanía que supondría la existencia de un

diálogo libre y abierto de todos los grupos socioculturales, lo que implica una política multicultural que incluya disposiciones de discriminación inversa, de intercambio etnocultural, y la prevención de desviaciones homogeneizadora o asimilacionista de la cultura hegemónica, en suma, el desafío de la interculturalidad. (Del Carpio, 2014, p. 128)

Esta multiculturalidad, al ofrecernos mayores perspectivas, una multiplicidad de miradas, nos da la oportunidad de consolidar nuestra humanidad, pues nos enriquece con miradas que no lograría obtener un hipotético parroquial europeo que se piense global o un 
posible provinciano conservadurista que se tema local. En efecto, esta opción de acceso a lo global y a lo local nos brinda una oportunidad emancipatoria, en la medida en que nos da una cierta libertad, aquella libertad - atrás mencionada - entendida como la articulación entre pertenencia y desasimiento.

Ahora bien, como decíamos anteriormente, la idea de los Derechos humanos es quizás el postulado que tiene una pretensión de alcance universal por antonomasia; sin embargo, es una idea que pretende democratizar las sociedades y pocos se oponen a su expansión y vigencia. Dentro de las competencias propuestas por el Ministerio de Educación Nacional se propone como competencia cognitiva en la que el ciudadano postule: "Conozco la Declaración Universal de los Derechos Humanos y su relación con los derechos fundamentales enunciados en la Constitución" (MEN, 2006). Competencia que resalta la importancia de los Derechos Humanos como institución democratizadora acogida por la ONU en 1948.

Sobre el mismo tópico de los Derechos Humanos, el Ministerio propone otra competencia cognitiva que propende por el entendimiento que la institución de los Derechos Humanos implica el derecho a la no discriminación. El texto plantea: "Comprendo que, según la Declaración Universal de los Derechos Humanos y la Constitución Nacional, las personas tenemos derecho a no ser discriminadas" (MEN, 2006). Igualmente, enseña que la Constitución Nacional también contiene y defiende este importante derecho a la no discriminación, derecho que está en el gozne que articula lo global -los derechos humanos-y lo local -las idiosincrasias múltiples que merecen respeto y protección.

Cuando el universo es más acotado, también podemos defender las particularidades, otros localismos, y en este sentido se orienta la competencia cognitiva-integradora propuesta por el Ministerio, esbozada así: "Analizo el manual de convivencia y las normas de mi institución;

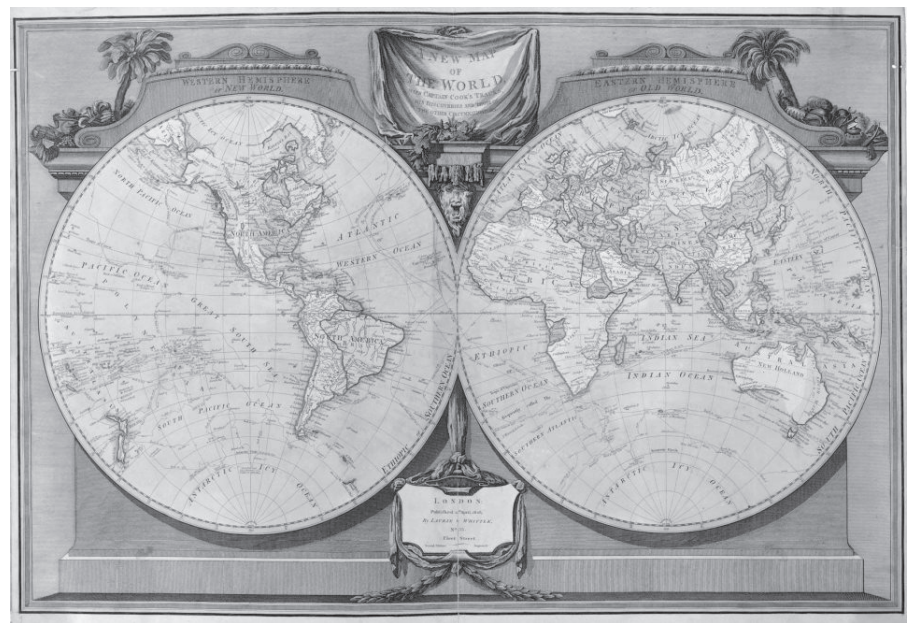

las cumplo voluntariamente y participo de manera pacífica en su transformación cuando las considero injustas" (MEN, 2006).

Dentro de las competencias cognitivas el Ministerio plantea la idea de la igualdad en la diversidad, idea muy importante que sería como la síntesis, la cifra de la disyuntiva entre derechos humanos y derechos de las autonomías; disyuntiva que es a su vez una síntesis ejemplificante de la dicotomía local-universal. El Ministerio plantea la mencionada competencia en estos términos: "Reconozco que los derechos se basan en la igualdad de los seres humanos, aunque cada uno sea, se exprese y viva de manera diferente" (MEN, 2006). Sin duda, es un aprendizaje necesario en la postmodernidad y en un país multicultural el reconocimiento de la igualdad dentro de las posibles diferencias idiosincrásicas del otro, pues es un aprendizaje forjador de sociedades democráticas.

El Ministerio propone otra competencia que puede servir como coadyuvante a la anterior; esta, en cambio, de carácter emocional y de propiedad integradora, que dice: "Manifiesto indignación (rechazo, dolor, rabia) cuando se vulneran las libertades de las personas y acudo a las autoridades apropiadas" (MEN, 2006).

Finalmente, dentro de las competencias comunicativas, el Ministerio señala también 
una competencia, que plantea: "Comprendo que el disenso y la discusión constructiva contribuyen al progreso del grupo" (MEN, 2006); competencia que reafirma la importancia de la existencia de disimilitudes, de disensos y de posturas particulares, que en una gestión constructiva pueden generar enriquecimiento del colectivo.

Ahora bien, puede resultar irónico que el planteamiento del Ministerio de Educación Nacional sobre el enfoque por competencias para el logro, afianzamiento y pervivencia de la riqueza multicultural, se base en un modelo foráneo que no fue producto de las vicisitudes propias de nuestra compleja nacionalidad. No obstante esta objeción, en conclusión podemos ver que mediante las competencias propuestas por el Ministerio de Educación Nacional se hace una aproximación, mediante destrezas comunicativas y emocionales, así como mediante aptitudes cognoscitivas, a expresiones de saberes locales y globales, que aunque de manera tímida, pueden ser un augurio de acceso a una postmodernidad multicultural.

\section{Referencias}

Constitución Política de Colombia. (1991). www.secretariasenado.gov.co

Del Carpio Rodríguez, C. (2014). Pluralismo jurídico, derecho humano a la identidad cultural y globalización. Madrid: Edit. Civitas:.

Delors, J. (1996). La Educación encierra un tesoro, Informe a la UNESCO de la Comisión Internacional sobre la Educación para el siglo XXI. Ediciones UNESCO, Santillana.

Dewey, J, (2014). Democracia y Educación. Madrid: Ediciones Morata.

Ferrer, E. (2009). Habermas: Universales sin absolutos. Por un diálogo constitucional mundial en aras de una búsqueda de la justicia sin fronteras. Revista A Parte Rei, 63.

Fischer, H. R., et al.,(1997). El Final de los Grandes Proyectos. Barcelona: Edit. Gedisa.

Geertz, C. (1994). Conocimiento local. Madrid: Ediciones Paidos Ibérica S.A.

Gergen, K. (1992). El Yo Saturado. Barcelona: Editorial Paidos.

Kymlicka, W. (2003). La Política Vernácula. Nacionalismo, multiculturalismo y ciudadanía. Madrid: Ediciones Paidós Ibérica S.A.

Kymlicka, W. (1996). Ciudadanía multicultural. Madrid: Ediciones Paidós Ibérica S.A.

Lipovetzky, G. (1986). La Era del Vacío. Barcelona: Anagrama.

Ministerio de Educación Nacional de Colombia (2006). Estándares Básicos de Competencias en Lenguaje, Matemáticas, Ciencias y Ciudadanas. Bogotá: Edit. Imprenta Nacional.

Ministerio de Educación, Cultura y Deporte, España. (2003). Marco Común Europeo de Referencia para las Lenguas: Aprendizaje, Enseñanza, Evaluación. Madrid.

Parekh, B. (2005). Repensando el multiculturalismo. Madrid: Edit. Itsmo.

Spinoza, B. (2005). Ethics. New York: Edit. Penguin Classics.

Vattimo, G. y otros. (1994). En torno a la Posmodernidad. Barcelona: Edit. Anthropos.

Wallerstein, I. (2004). Las incertidumbres del saber. Barcelona: Edit. Gedisa. 